\title{
Hirschsprung's disease in the newborn
}

\section{An 11-year experience}

\author{
Theodore Z. Polley, Jr., 2, and Arnold G. Coran' \\ ${ }^{1}$ Section of Pediatric Surgery, University of Michigan Medical School and \\ 2 Mott Children's Hospital, Ann Arbor, Michigan, USA
}

\begin{abstract}
The records of all patients with Hirschsprung's disease diagnosed and treated at our institution between 1 July 1974 and 31 August 1985 were reviewed. Of these 99 patients, $35(35 \%)$ presented and were diagnosed within the first 30 days of life and constitute the basis of the present study group. Twenty-two infants $(63 \%)$ had standard rectosigmoid disease with a male-to-female ratio of 2.2:1. Only one infant was premature. The spectrum of presenting signs included abdominal distension in $19(54 \%)$, failure to pass meconium within the first $48 \mathrm{~h}$ of life in $16(46 \%)$, "constipation" in $12(34 \%)$, and vomiting in $9(26 \%)$. Intestinal perforation was a presenting sign in 2 patients $(6 \%)$ and enterocolitis occurred preoperatively in $4(12 \%)$. Evaluation was facilitated by diagnostic barium enema in $60 \%$ of the patients. In those infants able to undergo elective evaluation, the definitive diagnosis was made by suction rectal biopsy, which was accurate in all cases. In addition to the high proportion of patients with long-segment disease (13 patients, 37\%), there was a significant incidence of associated congenital anomalies $(26 \%)$, including Down's syndrome in $5(14 \%)$. Thirty-three of the 35 patients have undergone definitive treatment using the endorectal pullthrough procedure, performed at an average age of 12 months, with no mortality related to the operation. In addition to highlighting the high incidence of congenital anomalies, the large proportion of neonates with long-segment disease, and the reliability of the diagnostic barium enema, this subgroup of patients with Hirschsprung's dis-
\end{abstract}

Offprint requests to: T. Z. Polley, Jr., Room F7516, Box 066, Mott Children's Hospital, Ann Arbor, MI 48109, USA ease emphasizes the special diagnostic and management considerations required in the newborn infant who presents with sepsis of unknown etiology, intestinal obstruction, or constipation. A high index of suspicion, liberal use of suction rectal biopsy, early leveling colostomy, and definitive treatment by endorectal pull-through are important in achieving $0 \%$ operative mortality and excellent functional results.

Key words: Hirschsprung's disease - Newborn Bowel obstruction newborn

\section{Introduction}

Hirschsprung's disease has assumed an important position in the differential diagnosis of intestinal obstruction in the newborn period, as has been emphasized in a recent review of neonatal intestinal obstruction [7], in contrast to earlier reports which listed intestinal atresias and stenoses followed by malrotation as the most common causes [6]. Klein et al. [7] found in their review that Hirschsprung's disease was second only to necrotizing enterocolitis as the etiology of newborn intestinal obstruction. The importance of making the diagnosis of Hirschsprung's disease in the newborn period is underscored by the lethality of enterocolitis and its complications in this age group [9]. Furthermore, the findings differentiating Hirschsprung's disease from other forms of intestinal obstruction may be so subtle that if the diagnosis is not suspected, it will lead to dangerous and unnecessary delay. The subgroup of neonates with Hirschsprung's disease is a significant 
percentage of the overall series recently reported from our institution [2] and forms the basis of this report.

\section{Materials and methods}

The records of all children with Hirschsprung's disease diagnosed and treated between 1 July 1974 and 31 August 1985 were reviewed. In 35 of these 99 patients, the diagnosis of Hirschsprung's disease was made within the first 30 days of life. The detailed clinical findings in these neonates represent the data for this review.

\section{Results}

Twenty-two infants $(63 \%)$ had standard rectosigmoid disease, while $13(37 \%)$ had long-segment disease. Of the patients with long-segment disease, 1 had disease extending to the descending colon, 3 to the splenic flexure, and 1 to the transverse colon. In 4 patients, the entire colon was involved. Three of the remaining 4 patients had disease extending well into the terminal ileum; one of them was born with only $70 \mathrm{~cm}$ of small bowel. Finally, in one patient the disease extended to within $20 \mathrm{~cm}$ of the ligament of Treitz. Twentyfour of the patients were male and 11 were female, representing a ratio of $2.2: 1$. The average age at presentation was 9.9 days, with 6 patients (17\%) presenting on the first day of life.

The patient population displayed the common spectrum of presenting signs as well as some unusual features. Nineteen infants $(54 \%)$ presented with abdominal distention and $16(46 \%)$ with failure to pass meconium within the first $48 \mathrm{~h}$ of life. Twelve (34\%) presented with constipation, and vomiting was a prominent symptom in $9(26 \%)$. Two patients, one with free intraperitoneal air and the other with cecal perforation and localized abscess, required immediate laparotomy. Only 4 (11\%) patients had enterocolitis preoperatively.

In 23 infants, the diagnosis was made by suction rectal biopsy. Of the 5 patients in whom the definitive diagnosis was based on full-thickness rectal biopsy, 4 had biopsies performed at other institutions. One of the remaining 7 patients was diagnosed during operation for free intraperitoneal air and 1 during laparotomy for a perforated viscus; the remaining 5 were diagnosed intraoperatively during emergency procedures for intestinal obstruction. Among those patients diagnosed by suction rectal biopsy, there were no false-negative, false-positive, or equivocal results. Although never relied upon alone for definitive diagnosis, barium enema demonstrated features diagnostic of Hirschsprung's disease in $60 \%$ of cases.
Associated congenital anomalies were present in $9(26 \%)$ of the neonates. Of these, $5(14 \%)$ had Down's syndrome, 3 had severe congenital heart disease, 1 had multiple renal anomalies, 1 had Hanhart's syndrome, 1 had hemophilia, and 1 presented with Smith-Lemli-Opitz syndrome. Four (11\%) infants had a positive family history for Hirschsprung's disease. There was only 1 premature infant (34 weeks' gestation) in this series.

Thirty-three of the 35 patients have undergone an endorectal pull-through procedure (ERPT) at an average age of 1 year. The 2 remaining patients expired prior to performance of definitive surgery, one with near-total intestinal aganglionosis and the other with multiple associated congenital anomalies. There has been no mortality among the patients who underwent ERPT.

\section{Discussion}

The interest in separating out neonates with Hirschsprung's disease relates not only to the increasing importance of the diagnosis in the newborn with intestinal obstruction, but also to the known increased mortality in this group of infants (Table 1). For example, in 1954 Klein and Scarborough $[8]$ described a $70 \%$ mortality in this age group, and Sieber and Giradny in 1957 [6] reported $40 \%$ mortality. More recently, in 1978, Grosfeld et al. [5] reported a $9 \%$ mortality, and in 1982 Davies [3] reported 13\%. The postoperative mortality in our series of 33 newborns following ERPT was 0\%. In our collective series of 99 patients with Hirschsprung's disease [12], all three patients who died were diagnosed in the newborn period and none of these deaths were due to postoperative complications. One patient died 1.5 months following ERPT from complications of severe congenital heart disease; the second was untreatable, with aganglionosis extending to with-

Table 1. Literature review of neonatal Hirschsprung's disease

\begin{tabular}{|c|c|c|c|c|}
\hline Series & $\begin{array}{l}\text { Mor- } \\
\text { tality }\end{array}$ & Male & $\begin{array}{l}\text { Long- } \\
\text { segment } \\
\text { disease }\end{array}$ & $\begin{array}{l}\text { Congenital } \\
\text { anomalies }\end{array}$ \\
\hline Klein and Scarborough [8] & $70 \%$ & & & \\
\hline Sieber and Giradny [15] & $40 \%$ & & & \\
\hline Grosfeld [5] & $9 \%$ & & & \\
\hline Davis [3] & $13 \%$ & & $0 \%$ & \\
\hline Lister [11] & $32 \%$ & & $8 \%$ & $16 \%$ \\
\hline Fraser and Wilkinson [4] & $33 \%$ & & $22 \%$ & \\
\hline Bowring and Kern [12] & $22 \%$ & & $26 \%$ & \\
\hline Polley (1985) [12] & $0 \%$ & $69 \%$ & $37 \%$ & $26 \%$ \\
\hline
\end{tabular}


in $20 \mathrm{~cm}$ of the ligament of Treitz, and the third had multiple congenital anomalies associated with the Smith-Lemli-Optiz syndrome, including severe central nervous anomalies and short bowel syndrome.

The male predominance ( 24 patients, $69 \%$ ) in our series is similar to that published by others $[9$, 12, 18]. Except for the study of Fraser and Wilkinson in 1967 [10], most series, like our own, report a low incidence of Hirschsprung's disease in premature infants. Specifically, this incidence is $3 \%$ ( 1 patient) in our series compared to $4 \%$ as reported by Bowring and Kern [11], 9\% by Lister [11], and $3 \%$ by Sieber [14]. The incidence of abdominal distention (54\%) and constipation (46\%) in our patients at the time of presentation is very similar to that seen in other series. Two of our patients presented with intestinal perforation, which is uncommon but similar in incidence to the $3.1 \%$ of 501 patients reviewed by Swenson et al. [18]. Forty-six percent of our patients presented with failure to pass meconium in the first $48 \mathrm{~h}$ of life. Since more than one-half of the neonates did pass meconium within this time period, this observation does not automatically rule of Hirschsprung's disease.

In $4(11 \%)$ of our newborns, enterocolitis was seen preoperatively. One of the infants with preoperative enterocolitis died from severe associated congenital anomalies but had no episodes of enterocolitis after ileostomy. The other 3 patients did not experience any additional episodes after either enterostomy or ERPT.

Review of the literature both supports and refutes the importance of the barium enema in the diagnosis of Hirschsprung's disease. Fraser and Wilkinson [4] reported that barium enema was diagnostic in $89 \%$ of cases, closely approximating the figure of $87 \%$ reported by Davies [3]. This is in contradistinction to the reports of Suzuki et al. [17] and Boley et al. [1]. In series, the barium enema was consistent with the diagnosis of Hirschsprung's disease (narrowed segment and/or barium retention after $24 \mathrm{~h}$ in $21(60 \%)$ of the newborns. For the definitive diagnosis of Hirschsprung's disease, however, we continue to rely on suction rectal biopsy and have found it to be $100 \%$ accurate with no false-positive or false-negative results. In cases where emergency laparotomy is required before the results of suction biopsy are available, seromuscular biopsies done intraoperatively are utilized to make the definitive diagnosis. Of course, in these cases it is very important that an experienced pathologist capable of making the diagnosis from a frozen section be available.
The percentage of patients presenting in the newborn period who also have long-segment disease has been reported to be as low as $0 \%$ [3] and $8 \%$ [11]. The incidence in our series of $37 \%$ (13 patients) is unusually high even when compared with the series of Bowring and Kern (26\%) [2] and Fraser and Wilkinson (22\%) [4]. This group of 13 newborns represents $65 \%$ of all patients with longsegment disease seen at our institution. Thus, our incidence of neonatal long-segment Hirschsprung's disease is twice that seen in the larger collective series. Of the 6 familial cases reported in our series [12], 4 children had a sibling who presented in the newborn period and 3 of these 4 had long-segment disease.

Leveling colostomy or enterostomy in the newborn period remains our initial approach to neonatal Hirschsprung's disease. Several series in the past have suggested that this may not be necessary. Shimm and Swenson [13] stressed rectal irrigations as an important form of therapy, and suggested that colostomy may be contraindicated in infants who are too ill to tolerate an operation. Leenders et al., in 1970 [10], provided an interesting perspective on the need for colostomy by comparing their precolostomy-era mortality of $40 \%$ prior to 1957 with a mortality of $23 \%$ in infants treated with colostomy as late as 1968 . In 1980, So et al. [16] reported 20 newborns who were treated with rectal irrigations, followed by an early pull-through procedure without colostomy and had satisfactory results. We continue to recommend an early leveling colostomy and feel that our mortality of $0 \%$ in this group of newborns strongly supports that recommendation.

This review confirms the importance of Hirschsprung's disease in the differential diagnosis of neonatal intestinal obstruction. The $0 \%$ mortality and excellent long-term results support the continued application of our management program, which involves early suction rectal biopsy in suspected cases followed by leveling enterostomy once the pathology report is available. Our series emphasizes the high incidence of associated anomalies, the significant percentage of long-segment disease, and the characteristic appearance of the barium enema, which contrast with other reports in the literature.

\section{References}

1. Boley SJ, Dinari G, Cohen MI (1978) Hirschsprung's disease in the newborn. Clinics Perinatol 5: 45-60

2. Bowring AC, Kern IB (1972) The management of Hirschsprung's disease in the neonate. Aust Paediatr $\mathrm{J} 8$ : $121-127$ 
3. Davies MRQ (1982) The newborn infant with intestinal aganglionosis. Afr J Surg 20: 119-128

4. Fraser GC, Wilkinson AW (1967) Neonatal Hirschsprung's disease. $\mathrm{Br}$ Med J 3:7-10

5. Grosfeld JL, Ballantine TVN, Csicsko JF (1978) A critical evaluation of the Duhamel operation for Hirschsprung's disease. Arch Surg 113: 454-460

6. Gross RE (1953) The surgery of infancy and childhood. WB Saunders, Philadelphia London

7. Klein MD, Coran AG, Wesley JR, Drongowski RA (1984) Hirschsprung's disease in the newborn. J Pediatr Surg 19: $370-374$

8. Klein RR, Scarborough RA (1954) Hirschsprung's disease in the newborn. Am J Surg 88: 6-16

9. Kleinhaus S, Boley SJ, Sheran M, Sieber WK (1979) Hirschsprung's disease: survey of the members of the surgical section of the American Academy of Pediatrics. J Pediatr Surg 14: 588-597

10. Leenders E, Sieber WK, Kiesewetter WB (1970) Hirschsprung's disease. Surg Clin North Am 50: 907-918

11. Lister J (1977) The man and the disease. R Coll Surg Edinburgh 22: $377-384$
12. Polley TZ Jr, Coran AG, Wesley JR (1985) A ten-year experience with ninety-two cases of Hirschsprung's disease. Ann Surg 202: 349-355

13. Shimm WKT, Swenson O (1966) Treatment of congenital megacolon in 50 infants. Pediatrics 38: 185-193

14. Sieber WK (1979) Hirschsprung's disease. In: Ravitch MM, Welch KJ, Benson CD (eds) Pediatric surgery. Year Book Medical Publishers, Chicago London, pp $1035-1054$

15. Sieber WK, Giradny BR (1957) Management of symptomatic aganglionic megacolon in early infancy. Arch Surg 75: 388-397

16. So HB, Schwartz DL, Becker J, Daum F (1980) Endorectal "pullthrough" without preliminary colostomy in neonates with Hirschsprung's disease. J Pediatr Surg 15: $470-471$

17. Suzuki H, White JJ, El Shafie M (1973) Nonoperative diagnosis of Hirschsprung's disease in neonates. Pediatrics 51: $188-191$

18. Swenson O, Sherman JO, Fisher JH (1973) Diagnosis of congenital megacolon: an analysis of 501 patients. J Pediatr Surg 8: 587-594 